\title{
Characteristics of Heart Rate Reduction with Resumption of Supine Position in the Postural Tachycardia Syndrome: Factors Influencing Recovery
}

\author{
Kenneth A. Mayuga, Christopher E. Gaw, Curtis Tatsuoka and Fetnat Fouad-Tarazi
}

Heart and Vascular Institute, Dept. of Cardiovascular Medicine, Cleveland Clinic, 9500 Euclid Ave, Desk J2-2, Cleveland, $\mathrm{OH} 44195$, USA

\begin{abstract}
Objectives: Postural orthostatic tachycardia syndrome (POTS), a heart rate (HR) rise with upright positioning, is dependent on autonomic influences. HR recovery (HR decrease after exercise cessation) is a measure of autonomic function. Characteristics of HR reduction during supine Recovery after head-up Tilt in POTS patients have not been elucidated.

Methods: 113 subjects (mean age 41.7 years, 86 female), diagnosed with POTS on head-up Tilt were analyzed. HR's were recorded during baseline supine position, 70-degree Tilt, and $20 \mathrm{sec}, 1 \mathrm{~min}$ and $2 \mathrm{~min}$ of supine Recovery. Percent HR reduction during Recovery was calculated.

Results: Baseline HR was $68.7 \pm 13.4$ bpm. Maximum HR during Tilt was $109 \pm 16.9$ bpm. Mean HR was $84.2 \pm 20$ bpm at $20 \mathrm{sec}, 78.5 \pm 18.9 \mathrm{bpm}$ at 1 -min, and $77.1 \pm 18.3 \mathrm{bpm}$ at $2 \mathrm{~min}$ of Recovery. Younger age and slower baseline HR were associated with greater HR reductions at $20 \mathrm{sec}(\mathrm{p}=0.006, \mathrm{p}=0.000$, respectively). Younger age, slower baseline HR and less time to achieve POTS were associated with greater HR reductions at $1 \mathrm{~min}(\mathrm{p}=0.025, \mathrm{p}=0.000, \mathrm{p}=0.000$, respectively) and at $2 \min (\mathrm{p}=0.004, \mathrm{p}=0.000, \mathrm{p}=0.000$, respectively). Gender and baseline blood pressures were not significant.

Conclusions: In POTS patients, HR quickly decreases upon resuming supine position. Younger age, slower baseline HR and less time to achieve POTS were associated with greater HR reductions during supine Recovery. Further study is needed to determine mechanisms, as well as analyze differences in symptoms or prognosis.
\end{abstract}

Keywords: Heart Rate Recovery, Postural Orthostatic Tachycardia Syndrome, Tilt Table Test, Syncope.

\section{INTRODUCTION}

Postural orthostatic tachycardia syndrome (POTS), a form of orthostatic intolerance, is associated with a myriad of symptoms including headache, nausea, fatigue, palpitations, near-syncope and syncope[1] and commonly affects daily activities[2]. POTS has been defined as a rise in heart rate (HR) by 30 -bpm or to 120 -bpm within the first $10 \mathrm{~min}$ of head-up Tilt[1, 2], though a phenomenon of 'Late' POTS with a HR rise after 10 mins has been described[3]. The autonomic nervous system, comprised of both sympathetic and vagal activity, plays a crucial role in the pathogenesis of POTS. A proposed classification schema divides the mechanisms into 2 categories[4]: the partial dysautonomic and hyperadrenergic forms. In the partial dysautonomic form, patients appear to have mild autonomic neuropathy where peripheral vasculature is unable to maintain adequate resistance to combat gravity leading to an increase in sympathetic output with an increase in HR and cardiac contractility to

*Address correspondence to this author at the Heart and Vascular Institute, Dept. of Cardiovascular Medicine, Cleveland Clinic, 9500 Euclid Ave, Desk J2-2, Cleveland, OH 44195, USA; Tel: 216-444-5828; Fax: 216-445-3102; E-mail: fouadtf@ccf.org maintain cerebral perfusion. In the hyperadrenergic form, which may be due to a point mutation inhibiting reuptake of norepinephrine from the synaptic cleft[5], there is a hyperadrenergic state resulting from an increased norepinephrine "spillover" in response to sympathetic stimuli.

Abnormal heart rate recovery (HRR) after exercise treadmill testing has been found to be a significant predictor for mortality in patients with coronary artery disease with the proposed mechanism being altered autonomic function[6, 7]. During the initiation of exercise, there is an initial withdrawal of vagal tone followed by a rise in sympathetic tone and, conversely, the cessation of exercise is accompanied by a gradual withdrawal of sympathetic tone and a re-activation of vagal tone. Altered autonomic function with a delayed reactivation of vagal tone during recovery is the proposed mechanism behind abnormal HRR. HRR after exercise treadmill testing has been defined as the difference between HR during peak exercise and HR at 1 min after exercise cessation, with an abnormal HRR defined as a $<12$ bpm reduction in HR at $1 \min [6]$.

Both HRR after exercise and HR increases in POTS are highly dependent on the balance between sympathetic and vagal tone, with alterations leading to "abnormal" HRs. 
However, the characteristics of HR reduction during the supine Recovery phase of the Tilt test in patients with POTS, along with factors affecting the HR decrease, have yet to be fully defined. As opposed to solely testing for a HR increase with Tilt, examining the HR reduction during Recovery may provide further insight into the autonomic imbalance present in POTS.

\section{MATERIALS AND METHODOLOGY:}

Data was analyzed from a prospectively collected registry-database in a study approved by the Institutional Review Board. Subjects who presented to the Cleveland Clinic from January to December 2007 for evaluation of symptoms of orthostatic intolerance and subsequently diagnosed with POTS on head-up Tilt test in the Syncope Clinic were included. No differentiation was made between primary or secondary POTS; patients may have had orthostatic intolerance as the lone medical problem or in conjunction with other potential causes of autonomic dysfunction such as diabetes. It is standard practice in our Syncope center to not stop the patient's medications prior to Tilt in order to obtain "real world" diagnostic information and to avoid problems with medication discontinuation especially if they are indicated for other diseases (e.g. angiotensin converting enzymeinhibitors and beta-blockers for coronary artery disease). No differentiation was made between 'Early' versus 'Late' POTS [3]; the HR increase by $30 \mathrm{bpm}$ or to $120 \mathrm{bpm}$ may have occurred within 10 min (Early) or after 10 min (Late) of Tilt. Since, in our experience, the response of blood pressure to tilt in POTS patients varies (e.g. no change, drop in systolic and/or diastolic pressure), patients with orthostatic hypotension during Tilt were included; however, patients with a vasovagal response were excluded. Patients with pacemakers, significant artifact or arrhythmias on ECG monitoring, missing data or discrepant data (1 patient) were excluded. At total of 113 patients (86 women) with ages ranging from 13 to 96 (mean 42) years old were included in the analysis.

Subjects underwent head-up Tilt testing according to the standard protocol at this institution: supine, 30-degree Tilt (2 min), 45-degree Tilt (2 min), 70-degree Tilt (45 min), and return to supine-Recovery (5 minutes). Isoproterenol was not used. Baseline blood pressure and HR were recorded via automated cuff. Continuous ECG-waveforms were recorded and reviewed via a Windaq system (DATAQ Instruments, Inc). HR's during Tilt and Recovery were calculated from the ECG waveforms. Maximum HR was defined as the maximum HR during the last 3 min of Tilt; the starting point for Recovery was identified as the time when the table began returning to the supine position from 70-degree tilt. Resumption of supine posture took approximately 9 sec. HR was measured at $20 \mathrm{sec}, 1 \mathrm{~min}$ and 2 min of Recovery. The dependent variable was defined as the HR reduction at each time point in Recovery compared to the maximum HR achieved during Tilt, expressed as a percentage: (maximum HR during Tilt - HR during Recovery) / maximum HR during Tilt.

Statistical analysis was carried out using multivariate regression. A two-sided $\mathrm{p}<0.05$ was considered significant. Diagnostic plots for residuals indicated reasonable model fit and no deviation from regression model assumptions. The effects on HR reduction during Recovery of age, gender, baseline HR, baseline systolic and diastolic blood pressure, and the time to achieve POTS (elapsed time until the criteria for POTS were met) were analyzed. Only age, baseline HR and time to achieve POTS were significant predictors of the HR reduction in Recovery and were therefore included for further analysis. Age, baseline HR and time to achieve POTS as well as 2-way interactions between these variables were analyzed at each Recovery time point. Interactions between baseline HR and time to achieve POTS showed a trend at 20 sec and 1 min of Recovery but were not significant ( $\mathrm{p}=$ 0.07 ); therefore, interactions were not included in the final analysis. Values are presented as mean \pm standard deviation.

\section{RESULTS}

Mean age was $41.7 \pm 16.7$ years old (Table 1). Baseline HR was $68.7 \pm 13.4 \mathrm{bpm}$. Maximum HR during Tilt was 109 $\pm 16.9 \mathrm{bpm}$. At $20 \mathrm{sec}$ of Recovery, mean HR was $84.2 \pm 20$ bpm, representing a 23\% decrease from maximum. At $1 \mathrm{~min}$ of Recovery, mean HR was $78.5 \pm 18.9$ bpm, a $28 \%$ decrease from maximum. At 2 min of Recovery, mean HR was $77.1 \pm$ $18.3 \mathrm{bpm}$, a $29 \%$ decrease from maximum.

Younger age and a slower baseline HR were associated with greater HR reduction at 20 sec of Recovery $(p=0.006$, $\mathrm{p}=0.000$, respectively) (Table 2 ). Time to POTS was borderline significant at $20 \mathrm{sec}$ of Recovery $(\mathrm{p}=0.062)$. At 1 min of Recovery, younger age, slower baseline HR and less time to achieve POTS were associated with greater HR reduction ( $\mathrm{p}=0.025, \mathrm{p}=0.000, \mathrm{p}=0.000$, respectively). At 2 min of Recovery, younger age, lower baseline HR and less time to achieve POTS were associated with greater HR reduction ( $\mathrm{p}=0.004, \mathrm{p}=0.000, \mathrm{p}=0.000$, respectively).

\section{DISCUSSION}

A slower baseline HR was associated with greater HR reduction during supine Recovery. The slower baseline HR may reflect a relatively higher baseline vagal tone. After removing the postural challenge (Tilt) with its resulting increased sympathetic tone, the resumption of supine position allows for the withdrawal of sympathetic output and a relative resurgence of vagal tone. Those with a higher vagal tone at baseline may therefore achieve greater reductions in HR after resuming supine position.

Younger age is associated with greater HR reduction during supine Recovery. This may be explained if the mechanism of POTS is different for younger versus older patients. Whereas in younger patients, POTS may be due to an imbalance of sympathetic versus vagal tone, older patients may be more prone to circulatory or perhaps more subtle autonomic neural dysfunction problems.

Less time to achieve POTS during Tilt was associated with greater HR reduction during Recovery. This may be explained by a relatively rapid response of a sensitive autonomic nervous system to postural challenge with a withdrawal of vagal tone and increase in sympathetic output, perhaps acting via receptors located centrally or in the cardiopulmonary area, as opposed to via peripheral baroreceptors. Subsequently, when the patient is returned to supine 
Table 1. Data at Baseline, Tilt and Recovery (Mean \pm Standard Deviation)

\begin{tabular}{|l|l|}
\hline & \\
\hline \hline Age & $41.7 \pm 16.7$ years old \\
\hline Gender & 27 male / 86 female \\
\hline Baseline HR & $68.7 \pm 13.4 \mathrm{bpm}$ \\
\hline Baseline systolic BP & $122.7 \pm 21.4 \mathrm{mmHg}$ \\
\hline Baseline diastolic BP & $70.9 \pm 9.7 \mathrm{mmHg}$ \\
\hline Maximum HR during Tilt & $109 \pm 16.9 \mathrm{bpm}$ \\
\hline Time to POTS during Tilt & $16.5 \pm 12.5 \mathrm{~min}$ \\
\hline $\begin{array}{l}\text { HR at } 20 \text { sec of Recovery } \\
\text { (\% change from maximum HR) }\end{array}$ & $\begin{array}{c}84.2 \pm 20 \mathrm{bpm} \\
(23 \% \text { decrease })\end{array}$ \\
\hline $\begin{array}{l}\text { HR at } 1 \text { min of Recovery } \\
\text { (\% change from maximum HR) }\end{array}$ & $\begin{array}{l}78.5 \pm 18.9 \mathrm{bpm} \\
(28.2 \% \text { decrease })\end{array}$ \\
\hline $\begin{array}{l}\text { HR at } 2 \text { min of Recovery } \\
\text { (\% change from maximum HR) }\end{array}$ & $\begin{array}{c}77.1 \pm 18.3 \mathrm{bpm} \\
(29.4 \% \text { decrease) }\end{array}$ \\
\hline
\end{tabular}

$\mathrm{HR}=$ heart rate, $\mathrm{BP}=$ blood pressure, $\mathrm{POTS}=$ postural orthostatic tachycardia syndrome

Table 2. Independent Variable (age, time to POTS, baseline HR) effects on Dependent Variable (\% Reduction in HR Compared with Maximum HR) at Different Times During Recovery

\begin{tabular}{|c|c|c|c|c|c|c|}
\hline & \multicolumn{2}{|c|}{20 sec } & \multicolumn{2}{c|}{ 1 min min } \\
\hline & Standardized Coefficient & p & Standardized Coefficient & p & Standardized Coefficient & p \\
\hline \hline Age & -0.246 & 0.006 & -0.175 & 0.025 & -0.223 \\
\hline Time to POTS & -0.164 & 0.062 & -0.287 & 0.000 & -0.285 \\
\hline Baseline HR & -0.361 & 0.000 & -0.530 & 0.000 & 0.004 \\
\hline
\end{tabular}

$\mathrm{HR}=$ heart rate, $\mathrm{POTS}=$ postural orthostatic tachycardia syndrome

position and the challenge is removed, the change in autonomic tone quickly reverts back to baseline.

The findings of this study may have clinical implications. The relatively rapid decrease in HR seen with resumption of supine posture can be useful in reassuring patients that once they lie down and elevate their legs, their symptoms are likely to quickly resolve. For young patients with a slow baseline HR, it may be that these patients are less symptomatic and have a better prognosis. These patients may respond to lifestyle modification (such as increasing salt and fluid intake) or to physical therapy (to promote circulatory re-adaptation) without the need for beta-blockers[8].

\section{Prior Studies}

HRR has been previously defined as the difference between the HR achieved with peak exercise and HR at $1 \mathrm{~min}$ after exercise cessation. An abnormal HRR, defined generally as a $<12 \mathrm{bpm}$ decrease in HR by $1 \mathrm{~min}[6]$, has been shown to identify those at higher risk of mortality; the mechanism proposed is an abnormal delay in reactivation of vagal tone. During exercise, there is an initial withdrawal of vagal tone, followed by an increase in sympathetic tone[9].
During normal recovery, it is generally believed that there is a sympathetic "spillover" effect where catecholamines may actually peak after the cessation of exercise; thus, the mechanism of $\mathrm{HR}$ slowing immediately post-exercise is thought to be due to the resurgence of vagal tone. The studied populations have mainly consisted of those undergoing stress testing for diagnosis of coronary artery disease where HRR has been shown to have prognostic value. In a study of 2428 adults undergoing symptom-limited exercise testing, Cole et al. [6] reported a twofold increase in risk of overall mortality in patients who exhibited an abnormal HRR. In a study of 5234 healthy subjects, abnormal HRR has been proposed as a marker for increased mortality even in the absence of traditional cardiac risk factors[10]. Panzer et al. [11] evaluated the relationship between impaired fasting glucose and abnormal HRR and found that the two factors were strongly associated. The proposed mechanism was that impaired fasting glucose, while not in the diabetic range, may already start to have adverse neuropathic effects; this would therefore have adverse consequences on the functioning of the autonomic nervous system[11].

In a study of the effect of short-term exercise training in 25 patients with POTS, Shibata et al. [12] found that after a 
3 month exercise regimen, HR was lower at any given oxygen uptake level, peak HR was similar, but HRR from exercise was faster after training than before training $(\mathrm{P}=0.036$ for training and 0.009 for interaction). They concluded that short-term exercise training improves physical fitness and cardiovascular responses during exercise in patients with POTS.

In a study of aerobic conditioning and POTS, Burkhardt et al. [13] analyzed the charts of 202 adolescents who underwent both cycle ergometer exercise testing and Tilt table testing for orthostatic intolerance. The authors reported that $2 / 3$ of patients were deconditioned as determined by peak oxygen uptake $<80 \%$ predicted without any difference between POTS patients and non-POTS patients, concluding that there was no clear connection between deconditioning and POTS. They reported that mean HR was $155 \mathrm{bpm}$ in POTS patients and $150 \mathrm{bpm}$ in non-POTS patients during heavy exercise but did not report peak HR. Reported HR at 1 min of recovery was $162 \mathrm{bpm}$ while $\mathrm{HR}$ at 5 min was 125 bpm for POTS patients versus $117 \mathrm{bpm}$ for non-POTS patients and concluded that HR recovery was slower for patients with POTS. However, standard definitions of abnormal HRR were not observed ( $<12$ bpm decrease by $1 \mathrm{~min}$ ).

HR characteristics after cessation of challenge (either exercise or postural challenge with their associated increases in sympathetic outflow) lend further insight into autonomic balance. However, it should be noted that HRR after treadmill exercise has been mainly studied with respect to coronary artery disease and therefore findings regarding mortality should not be deduced to occur in the POTS population as well. To our knowledge, no prior studies have reported the characteristics of HR reduction with resumption of supine position in the postural tachycardia syndrome.

\section{CONCLUSIONS}

Younger age, slower baseline HR and less time to achieve POTS were associated with greater HR reductions during supine Recovery. The relatively rapid decrease in HR upon resuming supine posture has significance when discussing alleviating measures (i.e. lying down) for patients with symptomatic POTS. Further study is needed to evaluate differences in symptoms and prognosis as well as to define possible mechanisms.

\section{LIMITATIONS}

The present study has several limitations. Firstly, symptoms were not evaluated; it may be that those with greater HR reductions during recovery have fewer or less severe symptoms. Likewise, response to therapy may also be different. Secondly, no differentiation was made between primary and secondary POTS. However, it is standard practice in our Syncope center to take secondary causes of POTS into account when determining treatment so no differentiation is made when POTS is diagnosed. Thirdly, medications were not stopped prior to Tilt testing. However, it is standard practice in our Syncope center to not stop the patient's medications prior to Tilt in order to obtain "real world" diagnostic information and to avoid problems with medication discontinuation especially if they are indicated for other diseases. Lastly, no differentiation was made between 'Early' and 'Late' POTS. However, a continuous variable (time to achieve POTS) was included in the analysis and was found to be significant.

\section{ABBREVIATIONS}

$\begin{array}{lll}\text { HR } & =\text { Heart rate } \\ \text { HRR }= & \text { Heart rate recovery } \\ \text { POTS }= & \text { Postural orthostatic tachycardia syndrome }\end{array}$

\section{CONFLICTS OF INTEREST}

Dr Fouad-Tarazi has received research or symposia support from AstraZeneca, Shire, Pfizer, Merck, Medtronic, Daxor and Chelsea.

\section{REFERENCES}

[1] Grubb BP, Kanjwal Y, Kosinski DJ. The postural tachycardia syndrome: A concise guide to diagnosis and management. J Cardiovasc Electrophysiol 2006; 17; 108-12.

[2] Medow MS, Stewart JM. The postural tachycardia syndrome. Cardiol Rev 2007; 15: 67-75.

[3] Mayuga KA, Butters KB, Fouad-Tarazi F. Early versus late postural tachycardia: A re-evaluation of a syndrome. Clin Auton Res 2008; 18; $155-7$.

[4] Grubb BP. Postural tachycardia syndrome. Circulation 2008; 117: 2814-7.

[5] Shannon JR, Flattem NL, Jordan J, et al. Orthostatic intolerance and tachycardia associated with norepinephrine-transporter deficiency. N Engl J Med 2000; 342: 541-9.

[6] Cole CR, Blackstone EH, Pashkow FJ, Snader CE, Lauer MS. Heart-rate recovery immediately after exercise as a predictor of mortality. N Engl J Med 1999; 341: 1351-7.

[7] Vivekananthan DP, Blackstone EH, Pothier CE, Lauer MS. Heart rate recovery after exercise is a predictor of mortality, independent of the angiographic severity of coronary disease. J Am Coll Cardiol 2003; 42: 831-8.

[8] Benarroch EE. Postural tachycardia syndrome: A heterogeneous and multifactorial disorder. Mayo Clin Proc 2012; 87(12): 1214-25.

[9] Pierpont GL, Stolpman DR, Gornick CC. Heart rate recovery postexercise as an index of parasympathetic activity. J Auton Nerv Syst 2000; 80: 169-74.

[10] Cole CR, Foody JM, Blackstone EH, Lauer MS. Heart rate recovery after submaximal exercise testing as a predictor of mortality in a cardiovascularly healthy cohort. Ann Intern Med 2000; 132: 5525.

[11] Panzer C, Lauer MS, Brieke A, Blackstone E, Hoogwerf B. Association of fasting plasma glucose with heart rate recovery in healthy adults: A population-based study. Diabetes 2002; 51: 803-7.

[12] Shibata S, Fu Q, Bivens TB, Hastings JL, Wang W, Levine BD. Short-term exercise training improves the cardiovascular response to exercise in the postural orthostatic tachycardia syndrome. J Physiol 2012 (15): 3495-505.

[13] Burkhardt BE, Fischer PR, Brands CK, et al. Exercise performance in adolescents with autonomic dysfunction. J Pediatr 2011; 158: $15-9$. 